\title{
Intra-median raphe nucleus (MRN) infusions of muscimol, a GABA-A receptor agonist, reinstate alcohol seeking in rats: role of impulsivity and reward
}

Anh Dzung Lê • Douglas Funk • Stephen Harding •

Walter Juzytsch • Zhaoxia Li • Paul J. Fletcher

Published online: 13 November 2007

(C) Springer-Verlag 2007

In this article the senior author's name was incorrectly specified in the list of authors and in the affiliation list. It should read Anh Dzung Lê.

The online version of the original article can be found at http://dx.doi. org/10.1007/s00213-007-0943-4.

A. Dzung Lê $(\bowtie) \cdot$ D. Funk $\cdot S$. Harding $\cdot$ W. Juzytsch $\cdot$

Z. Li $\cdot$ P. J. Fletcher

Department of Neuroscience,

Centre for Addiction and Mental Health,

33 Russell Street,

Toronto, ON M5S 2S1, Canada

e-mail: Anh_Le@camh.net

A. Dzung Lê

Department of Pharmacology, University of Toronto,

Toronto, Canada

A. Dzung Lê · P. J. Fletcher

Department of Psychiatry, University of Toronto,

Toronto, Canada

P. J. Fletcher

Department of Psychology, University of Toronto,

Toronto, Canada 